\title{
Evaluating the Effectiveness of Emotion Recognition Software as an Interactional Tool for Individuals with Autism: An Autism Caregiver/Professional Perspective
}

\author{
Aryan Dawer \\ Modern School Barakhamba Road, New Delhi, Delhi, 110001, INDIA; aryandawer7@icloud.com
}

\begin{abstract}
The aim of this research study was to evaluate the impact of a self-formulated video on a self-designed Emotion Recognition Software (ERS) on autism professionals' or family members' perceptions of the effectiveness of ERS. ERS is a tool for enabling people with Autism Spectrum Disorder (ASD) to interpret emotions in daily interactions. In the study, the respondents were given a brief description of the software and asked to rate its effectiveness. Then they were shown a video explaining and displaying the working of the ERS. Out of the seven emotions shown, they were asked to rate the emotions (on a " $1-7$ " scale) on the basis of their perceptions of the software's efficacy at interpreting each of them and asked to give the software an overall rating. The results from the paired t-test revealed that the video was able to change the respondents' perceptions (mean rating changed from 4.8 to 5.5 ) and after watching the video, less than 3.5\% of the respondents rated the ERS a "3 or below" (as opposed to 17.5\% before) and more than $82 \%$ rated the ERS a " 5 " or above" (as opposed to $65.5 \%$ before). Interestingly, the results showed that only emotions of happiness and fear affected the respondents' final perceptions of the software. The support shown for the software in the quantitative and qualitative data, after watching the video and understanding its functionality and benefits, suggests that people are excited about its use in real life.
\end{abstract}

KEYWORDS: Behavioural and Social Sciences; Social Psychology; Emotion Recognition Software; Autism Spectrum Disorder; Efficacy Analysis.

\section{- Introduction}

For most of us who engage in social interactions naturally and spontaneously, a striking feature of autistic individuals is their struggle with affective contact. ${ }^{1}$ This means that a person with autism, a condition known formally as the Autism Spectrum Disorder (ASD), is likely to be fascinated with objects, but be indifferent to people, which can come across as being off-putting for most people. An anecdote shared by "Jenny", a mother of her autistic child, who visited her psychology professor at the latter's office, provides a quintessential example of this characteristic. During this office visit, Jenny's then two-year-old autistic son never once looked at the professor. Instead, he stepped all over her couch swinging all her canvases, lost in their swinging motion, utterly uninterested in any human presence (personal communication, May 5, 2021).

Relatedly, ASD is characterized by impairments in social interaction due to deficits and/or atypical features in the areas of verbal and non-verbal communication, as well as difficulties with reciprocating at a social and emotional level. ${ }^{2}$ Therefore, autistic individuals find social interactions to be a great source of stress and anxiety, which can make their lives particularly challenging in mainstream society.

It is important to point out that social interactions can be difficult, even in the case of mildly autistic individuals who possess verbal skills of communication and often function independently in mainstream society, due to aspects of non-verbal communication such as facial expressions, gestures, and eye contact. Several studies describe individuals with ASD as be- ing unable to recognize mental and emotional states in others and in themselves. ${ }^{3,4} \mathrm{~A}$ possible reason is that individuals with ASD are themselves lacking in facial expressions: they do not display their emotions outwardly through facial expressions. ${ }^{1}$ For instance, when individuals with ASD are happy or angry, they do not display discernible conventional facial expressions of happiness or anger. This could make it difficult for them to read these expressions in others and to be able to assign meaning and intentions to the conversation partners. Other studies have shown that individuals with ASD tend to look less at the eye region of the face, ${ }^{5,6}$ engage in less mutual eye gaze behavior, ${ }^{7}$ and struggle to follow the gaze, ${ }^{8}$ compared to neurotypical individuals. Such a difficulty could thus further add to their sense of anxiety about social interactions.

Since the human face is central in both the expression and communication of emotion, researchers have sought to investigate ASD individuals' ability to identify emotions. Some studies have found children and adults with high-functioning autism (HFA) can recognize basic emotions, such as the six basic universally recognized emotions (happiness, sadness, fear, anger, surprise, and disgust), from pictures and that they only have problems recognizing "complex emotions" (such as embarrassment, insincerity, intimacy, etc.) in both adults and children with ASD. ${ }^{4,10}$ However, the ASD spectrum is vast and we need to look for ways to help those on the low-functioning end as well. It is also debatable if pictures can truly capture various portraits of emotions. There is a need for more sophisticated 
tools either to train autistic individuals in emotion recognition or simply do it for them.

A solution that could address this challenge faced by individuals with ASD is in the development and application of computational tools that enable emotion recognition through facial expressions. ${ }^{11,14}$ An ERS is designed to interpret emotions based on facial expressions identified through a video feed, which are transmitted to be processed in the program in real time. It categorizes facial expressions under one of seven emotions: anger, disgust, fear, happiness, sadness, surprise, and neutral. The software has been trained through the use of a neural network by showing images of different people displaying different emotions. This tool has become an ideal way to support individuals with ASD on the entire spectrum. ${ }^{10}$

Several studies have been done using computational tools to try and help individuals with ASD and to measure the efficiency and validity of these tools. For instance, in one study, two groups of 11 children (aged 12-18) with autism participated: one group used the computer program for 10 half-hour sessions over 2 weeks, the other didn't. ${ }^{21}$ Within-program data showed a significant reduction in errors made from first to last use. Students were assessed pre- and post-intervention using facial expression photographs, cartoons depicting emotionladen situations, and non-literal stories. Scores were not related to age or verbal ability. The experimental group made gains relative to the control group on all three measures. Gains correlated significantly with the number of times the computer program was used and results suggest positive effects.

Stanford University's Autism Glass Project uses Google's face-worn computing system to aid autism-affected subjects in understanding appropriate social cues by appending emoticons to those expressions the system is able to recognize (Figure 1). ${ }^{16}$

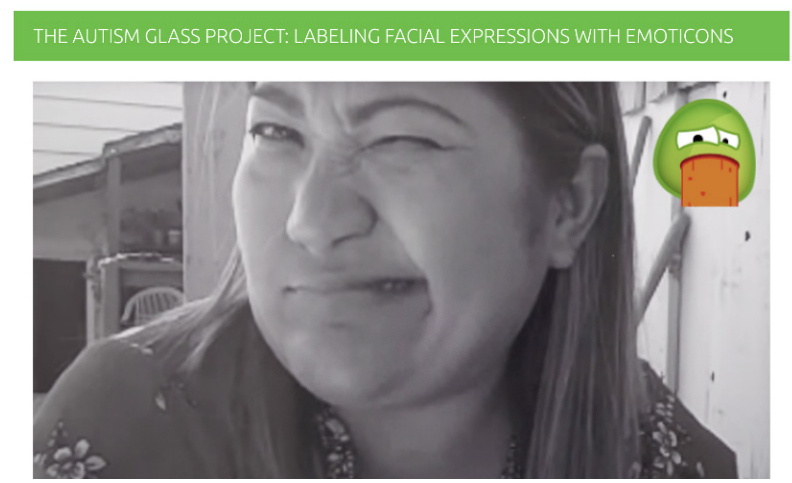

Figure 1: Stanford University's Autism Glass Project.

Note: A display of emotion recognition through emoticons. Reprinted from The Future of Emotion Recognition in Machine Learning. In Iflexicon blog, November 20, 2020, from https://www.iflexion.com/blog/emotion-recognition-software.

The usefulness of ERP tools could thus be tremendous for people with ASD by reducing the aspects of social interactions, which they need to worry about in a conversation. Oftentimes, these programs contain biases not only in the recognition of the emotions themselves but also based on whose emotions are being interpreted. For example, a man and a woman exhibiting the same emotion would be interpreted differently. However, there are concerns regarding the application of this technology in real-life situations. Since it is impossible to guarantee the $100 \%$ accuracy of a software that works on a pre-trained model, an autistic person could become unduly distressed by the wrong judgment of the software.

Therefore, to contribute to the evaluation of the perceived pros and cons of using an ERS for ASD individuals, this research study to harness the perceptions of autism professionals or family members regarding this topic by showing them a video created as a part of this research. This video shows a self-designed ERS at work in interpreting the seven common emotions. Since autism professionals and people related to ASD loved ones have first-hand experiences with ASD individuals and ASD individuals' interaction needs, their opinions on the viability of this software could be invaluable.

\section{- Methods}

\section{Research Aim and Research Approach:}

The aim of this research study was to evaluate the impact of a self-formulated video on a self-designed ERS on the perceptions of autism professionals and family members towards the effectiveness of ERS. This study's goal also included examining the ERS in general as a tool for enabling ASD individuals to interpret emotions in daily interactions. More specifically, the respondents were shown the ERS in action, through a video in order to evaluate the efficacy of the software to interpret each emotion.

The hypotheses were as follows:

1a. Null Hypothesis: There is no difference between the mean ratings of the respondents' evaluations of the ERS' efficacy in interpreting the different emotions.

1b. Alternative Hypothesis: There are differences between the mean ratings of the respondents' evaluations of the ERS' efficacy in interpreting the different emotions.

2a. Null Hypothesis: The video has no effect on the respondents' opinions on the effectiveness of ERS for people with ASD.

2b. Alternative Hypothesis: The video has an effect on respondents' opinions on the effectiveness of the ERS for people with ASD.

3a. Null Hypothesis: Respondents' evaluations of the ERS' efficacy in interpreting the different emotions has no effect on their overall rating of its effectiveness for ASD individuals.

3b. Alternate Hypothesis: Respondents' evaluations of the ERS' efficacy in interpreting the different emotions has an effect on their overall rating of its effectiveness for ASD individuals.

\section{Data Collection:}

The data collection for this research study consisted of two steps:

1) Design of the Software

2) Design of the Questionnaire

\section{Design of the Software:}

The emotional recognition software is a Machine Learning model (MLM) trained on the FER-2013 dataset that consists of 28,709 48x48-pixel training images. The software works on real-time video and identifies emotions by categorizing facial expressions under one of seven emotions: 
works on real-time video and identifies emotions by categorizing facial expressions under one of seven emotions (Figure2):

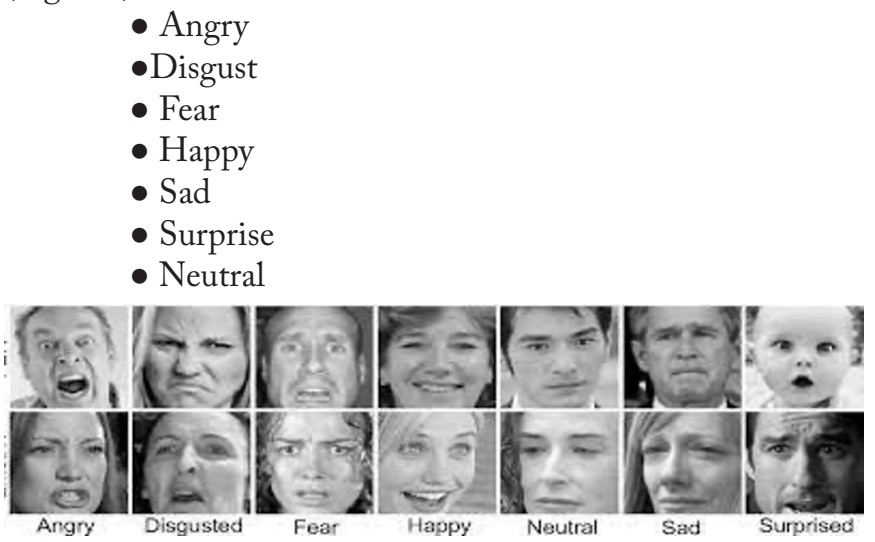

Figure 2: FER-2013 dataset display.

Note: FER-2013 Learn Facial Expressions from an Image. Kaggle, 2020 https://www.kaggle.com/msambare/fer2013

In the neural network model, a Convolutional Neural Network architecture was followed. Specifically, the program divided an image into different sections and examined them separately to find common features in particular sections of multiple images. ${ }^{17}$ For instance, the section focused on one's eyes may show tension in the eyebrows while exhibiting the emotion of anger. The number of dropout layers were increased in this study. Dropout layers, as the name suggests, drop some pixels of the image by a customizable factor, for example, 115 pixels of a $48 \times 48$ pixel image (roughly $0.05 \%$ ) while training the model. ${ }^{18}$ This ensured that the model did not get accustomed to seeing the same portions of different pictures. For instance, if the program was identifying anger by examining the tension in one's eyebrows, a person who exhibits anger differently cannot be interpreted correctly. To avoid such a situation, and to prevent any bias in the recognition process, the dropout layers were increased, a very creative idea given that the users of the tool themselves do not have the ability to exhibit emotions through facial expressions.

\section{Design of the Questionnaire:}

A questionnaire was designed, which comprised three main sections:

- Respondents' relation to an individual with ASD (professional, parent or sibling)

- Close-ended questions to evaluate respondents' perceptions towards emotional recognition software,

- Before showing the video, the respondents were informed about the function and power of the ERS and asked to assess their perceptions of its effectiveness in helping people with ASD by giving it a rating on a scale of 1 to 7 .

- Then the self-prepared video was shown: https://youtu.be/ WjKTnTR7EB4

- Then after watching the video, the respondents were asked to rate, on a scale of one to seven, how well the ERS did in in identifying the following emotions: anger, disgust, fear, happiness, sadness, surprise, neutrality.

- Respondents were asked if they think that ERSs could help people with ASD in their daily interactions and rate the software on a scale of 1 to 7 again.
- Respondents were asked if they think that ERSs could help people with ASD in their daily interactions and rate the software on a scale of 1 to 7 again.

- Open-ended questions to evaluate the respondents' perceptions towards ERS. Respondents were asked to explain their ratings on various questions and give suggestions for improvement in the software. experience of contact by applying force, vibrations, or motions to the user.

An invitation letter was prepared to be sent with the questionnaire, giving background information about the author of the research study. The form was sent to various organizations and support groups for people on the autism spectrum. It was also posted on social media autism communities such as Facebook groups with parents of children with ASD to get a diverse sample group of respondents with unique stories and experiences. Subsequently, the survey was filled by 29 people: 17 parents, one sibling, and 11 professionals.

\section{Data Analysis:}

For the analysis of the data, descriptive statistics were used to determine the mean differences between the respondents' ratings. Moreover, a one-way ANOVA test was to evaluate the statistical significance of the mean differences between the ratings that the respondents gave to the efficacy of the ERS to identify each emotion. Next, in order to compare the ratings given by the respondent to the viability of the ERS before and after watching the video, a Paired t-Test was run on the data. After that, a multiple regression analysis was conducted on the group to evaluate the impact of the efficacy of the machine to identify the seven emotions on the respondents' likely overall rating to the viability of the ERS. Then, once the important features were identified, it was run again on them to calculate the percentage of their importance and their coefficients in the equation to predict the respondent's overall rating of the ERS. The responses were graphed on a bar chart to show a clearer picture of the distribution of the proportion of the respondents across the ratings. Finally, qualitative data were analyzed based on the respondents' answers to an open-ended question, asking them to elaborate upon the factors influencing their ratings and suggestions to improve the program.

\section{- Results and Discussion}

The respondents' ratings of the efficacy of the self-designed facial ERS in recognizing seven basic universal emotions and their evaluation of the viability of the program for ASD individuals before and after watching the video were compared. Moreover, their responses to open-ended questions elicited additional perspectives and suggestions for the improvement in the aforementioned software.

\section{Efficiency in Interpreting Emotions:}

Descriptive statistics show that the emotion "Happiness" (Figure 3) $(M=6.21, S D=1.11)$ had the highest rating, followed by "Sadness" $(M=5.86, S D=1.36)$, "Anger" $(M=5.48$, $S D=1.35)$, "Surprise" $(M=5.31, S D=1.56)$, "Fear" $(M=5.24$, $S D=1.38)$, "Neutrality" $(M=5.07, S D=1.65)$, and finally Disgust $(M=4.79, S D=1.61)$ (see Table 1$)$. It is evident that the standard deviations within the responses of each emotion are almost the same, which suggest that the people's ratings of the efficacy of the program were very similar. Given a scale of 
to seven, the ratings given to the program's efficacy $(M=5.43)$ to interpret various emotions were very impressive.

Table 1: Descriptive Statistics - Comparison of Ratings of Efficiency in Interpreting Emotions.

\begin{tabular}{|l|r|r|r|r|r|}
\hline \multicolumn{1}{|c|}{ Groups } & Count & Sum & \multicolumn{1}{c|}{ Average } & \multicolumn{1}{c|}{ Variance } & \multicolumn{1}{c|}{$\begin{array}{l}\text { Standard } \\
\text { Deviation }\end{array}$} \\
\hline Anger & 29 & 159 & 5.482758621 & 1.830049261 & 1.352793133 \\
\hline Disgust & 29 & 139 & 4.793103448 & 2.598522167 & 1.611993228 \\
\hline Fear & 29 & 152 & 5.24137931 & 1.903940887 & 1.379833645 \\
\hline Happiness & 29 & 180 & 6.20689655 & 1.24137931 & 1.114172029 \\
\hline Sadness & 29 & 170 & 5.862068966 & 1.837438424 & 1.355521458 \\
\hline Surprise & 29 & 154 & 5.310344828 & 2.435960591 & 1.560756416 \\
\hline Neutrality & 29 & 147 & 5.068965517 & 2.709359606 & 1.646013246 \\
\hline
\end{tabular}

To determine whether the mean differences were statistically significant, a one-way ANOVA was run. The one-way ANOVA for emotions shows statistical significance: $\mathrm{F}(6,196)$ $=3.20$ (higher than the $\mathrm{F}$ critical value of 2.15), $\mathrm{p}<.01$ (see Table 2).

Table 2: One-Way ANOVA - Comparison of Ratings of Efficiency in Interpreting Emotions. P-value less than 0.01 and $\mathrm{F}$ is more than F-critical showing statistical significance of results.

\begin{tabular}{|l|c|r|c|c|c|c|}
\hline \multicolumn{1}{|c|}{$\begin{array}{c}\text { Source of } \\
\text { Variation }\end{array}$} & \multicolumn{1}{c|}{ SS } & \multicolumn{1}{c|}{$\boldsymbol{d f}$} & \multicolumn{1}{c|}{ MS } & $\boldsymbol{F}$ & P-value & $\boldsymbol{F}$ crit \\
\hline Between Groups & 39.98029557 & 6 & 6.663382594 & 3.20428652 & 0.0050451635 & 2.145071468 \\
\hline Within Groups & 407.5862069 & 196 & 2.079521464 & & & \\
\hline & & & & & & \\
\hline Total & 447.5665025 & 202 & & & & \\
\hline
\end{tabular}

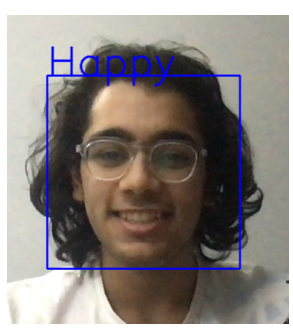

Figure 3: ERS Recognizing the Emotion of Happinesss.

Note: ERP EXPLAINED. Youtube, AD 007, Apr 26, 2021 https://www.youtube.com/watch?v=WjKTnTR7EB4

However, disgust (4.79), a more nuanced and complex emotion, was rated the lowest(Figure 4). Qualitative data offered important insights as to the complexity of guaranteeing the optimal efficacy of the ERS, which is so vital for ASD individuals. First of all, some facial expressions are too ambiguous to predict. Respondents described the emotions as being "too similar", "tricky to predict", and "can overlap". This means that even if the software can detect them correctly, one can never know for sure that it is the intended emotion of the other person because the facial expressions and features used by the ERS to recognize the emotion could be associated with other emotions as well.

Second, people express their emotions through different facial expressions, as stated by one of the survey respondents: "Not everyone shows emotions in the same way." This means that different people have different facial expressions even when they are exhibiting the same emotion. However, this concern of the respondents should have already been accounted for by the usage of FER-2013 dataset in training the model for the software. The FER-2013 dataset included expressions of people of different genders, races, and ethnicities to ensure that precise prediction doesn't only occur for one group of people. Instead, the software was able to categorize different expressions to interpret emotions in people on the basis of the aforementioned factors. However, one can never confirm that every person's way of expressing a particular emotion has been accounted for.

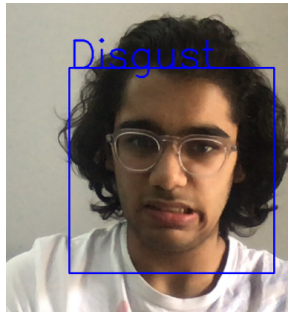

Figure 4: ERS Recognizing the Emotion of Disgust.

Note: ERP EXPLAINED. Youtube, AD 007, Apr 26, 2021

https://www.youtube.com/watch?v=WjKTnTR7EB4

Finally, the fundamental lack of facial affect in individuals with ASD, i.e., their inability to express emotions in their facial expressions means that it may still be cognitively challenging for ASD individuals to use this information to predict emotion, which was pointed out by one of the respondents: "I think the ERP is useful to help ASD individuals recognize facial emotions. However, it doesn't help them to understand WHAT is that emotion or feeling." This statement suggested that the respondents' concern about the use of the ERS does not lie so much with the efficacy of the program, but with the ASD individuals' neurological ability to actually understand emotions. Therefore, they questioned whether the software would be of any use to the ASD individuals in interactions. Nonetheless, most of the respondents showing such a concern were still ready to "give it a shot".

The distribution of the respondents across the ratings on each of the seven emotions was further examined and compared (see Figures 5-11). It was seen that the emotions of happiness and sadness have around $75 \%$ of the respondents rating it " 6 " or above, whereas the more ambiguous emotions such as neutrality, anger and disgust are rated a " 5 " or " 6 " by almost $55 \%$ of the respondents, thus validating the statistical results that the program performed better with these emotions from the perspective of the respondents.

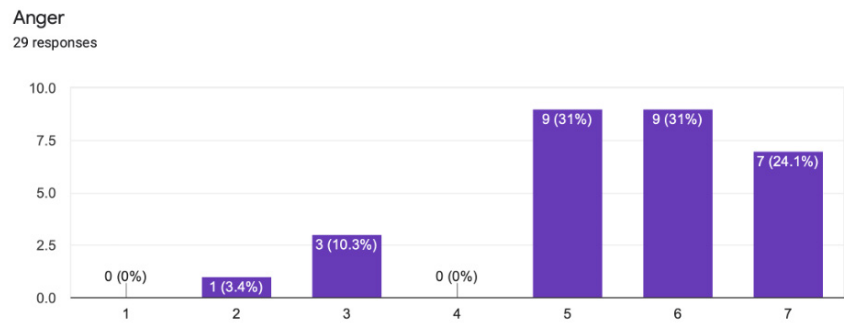

Figure 5: ERS Recognizing the Emotion of Happinesss. 


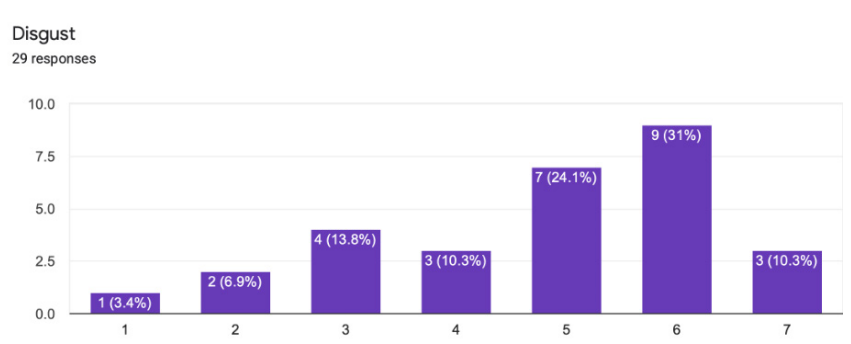

Figure 6: Ratings of Efficiency in Interpreting Disgust.

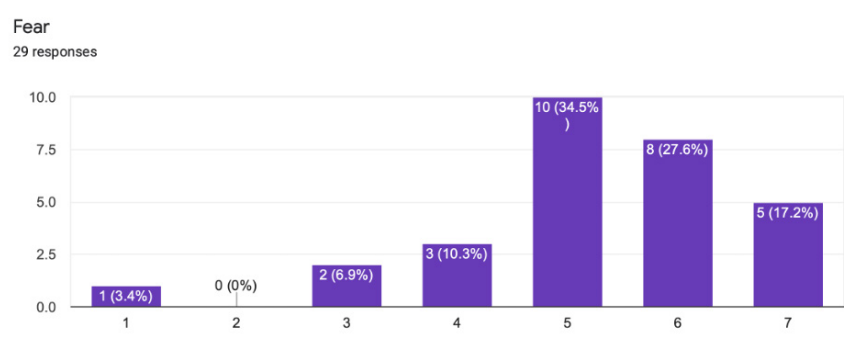

Figure 7: Ratings of Efficiency in Interpreting Fear.

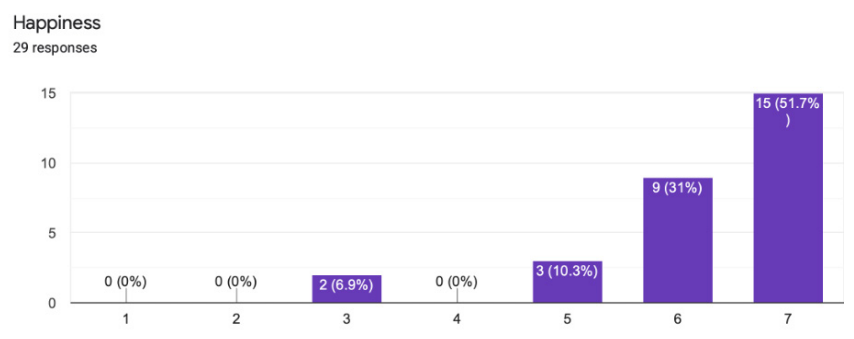

Figure 8: Ratings of Efficiency in Interpreting Happiness.

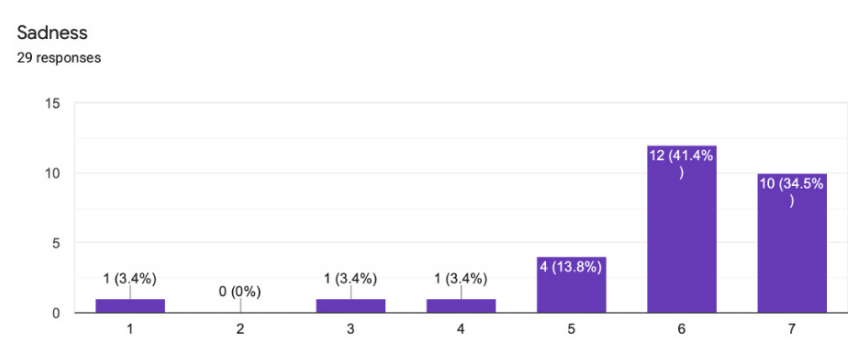

Figure 9: Ratings of Efficiency in Interpreting Sadness.

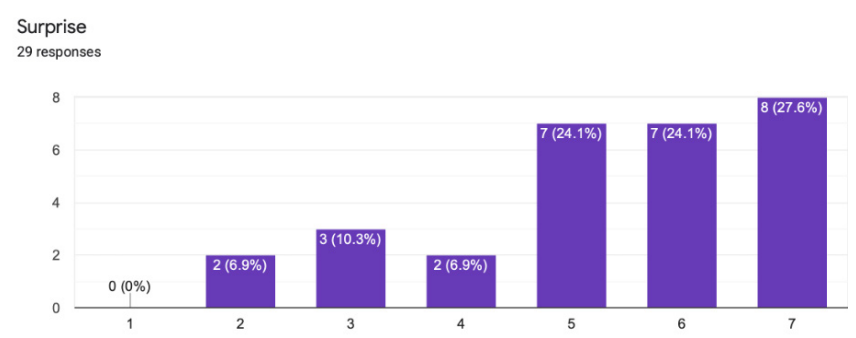

Figure 10: Ratings of Efficiency in Interpreting Surprise.

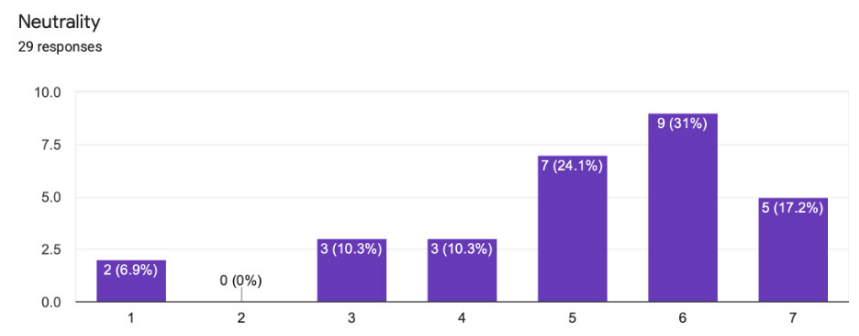

Figure 11: Ratings of Efficiency in Interpreting Neutrality.
Respondents' Perceptions of the Effectiveness of ERS for ASD Individuals:

A paired t-test was run to determine whether the mean difference between the respondents' perceptions and ratings before and after watching the video was statistically significant. Table 3 shows that the mean difference between the ratings was statistically significant, $t(28)=2.81$ [more than the $t$ critical value of 2.05 (two-tailed)], $p<.01$ (two-tailed). This indicates that the respondents showed a statistically significant change in ratings of effectiveness of the ERS upon watching the video, understanding the concept a little better, and seeing the emotions being interpreted.

Table 3: Respondents' Perception of Effectiveness of ERS for ASD Individuals (Before vs. After). P-value less than 0.01 and $t$-stat is more than t-critical showing statistical significance of results.

\begin{tabular}{|l|r|r|}
\hline \multicolumn{3}{|c|}{ t-Test: Paired Two Sample for Means } \\
\hline & Effectiveness (After) & Effectiveness (Before) \\
\hline Mean & 5.482758621 & 4.75862069 \\
\hline Variance & 1.25862069 & 1.475369458 \\
\hline Observations & 29 & 29 \\
\hline Pearson Correlation & 0.298236011730524 & \\
\hline Hypothesized Mean & & \\
Difference & 0 & \\
\hline df & 28 & \\
\hline t Stat & 2.813429334 & \\
\hline P(T<=t) one-tail & 0.004431709 & \\
\hline C Critical one-tail & 1.701130934 & \\
\hline P(T<=t) two-tail & 0.008863417 & \\
\hline t Critical two-tail & 2.048407142 & \\
\hline
\end{tabular}

The fact that the respondents showed a statistically significant change in perception is further examined by looking in the graphs (see Figures 12 and 13). Before watching the video, around $35 \%$ of the respondents had rated the effectiveness of ERS as a tool for ASD individuals in the range of 1-4 out of 7. However, after watching the video, this percentage halved to become around $17 \%$.

Based on your current knowledge of the ERP, how would you rate the effectiveness of ERPs (in general) in helping ASD individuals interpret emotion...ot effective at all" and "7" = "Highly effective") ? 29 responses

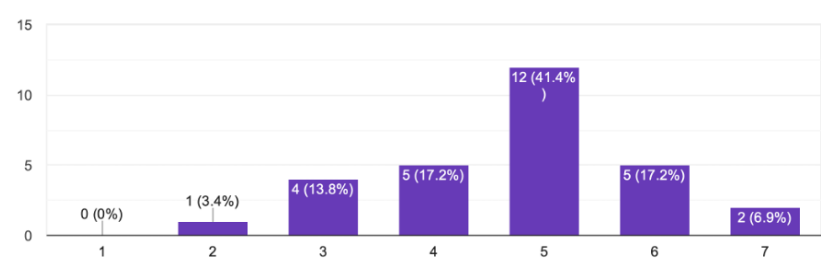

Figure 12: Bar Graph showing Respondents' Rating on the Viability of the Software Before watching the Video.

Based on this video, how would you rate the effectiveness of ERPs (in general) in helping ASD individuals to interpret the emotions in their daily e..."Not effective at all" and "7" = "Highly effective") ? 29 responses

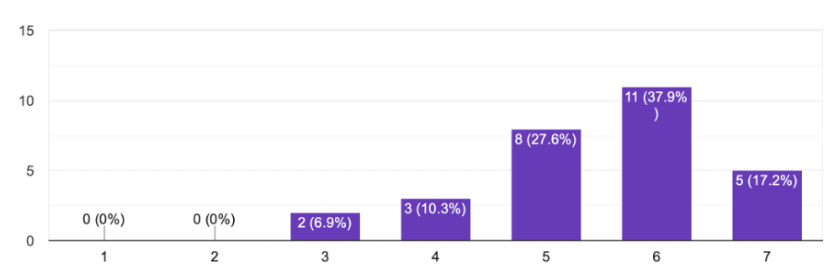

Figure 13: Bar Graph showing Respondents' Rating on the Viability of the Software Before watching the Video. Note the change in median rating from 5 to 6 which shows the effectiveness of the video. 
It is also important to note that with the responses after watching the video, there wasn't a single respondent who rated the program a " 1 " or a " 2 " and more than $82 \%$ rated it a "5 or higher". This data indicates that every respondent, on some level, did not dismiss the possibility that ERS could be effective as a tool for ASD individuals. The enthusiasm about this technology, after watching the video, was exhibited through the responses to open-ended questions as well. Respondents were excited about various aspects. Some appreciated the working principle of the software, as in the case of one parent who stated, "My son definitely learns best with pictures. It has helped him tremendously with recognizing different emotions". This highlights the fact that visual media may not only be the best way for learning for autistic individuals but might also be the most comfortable and adaptive medium.

Identification of Ratings of Individual Emotions Impacting Rating of Effectiveness of ERS for ASD Individuals and Extent of Impact:

A multiple regression analysis was conducted to predict the effects of the respondents' ratings of the influencing factors on their ratings of the effectiveness of ERS for ASD individuals after watching the video. Of the seven factors, happiness and fear were found to be statistically significant (see Table 4): Happiness, $b=0.70, \mathrm{t}(21)=3.20$ (higher than the critical value of 2.09), $p<.01$; and Fear, $b=0.57, t(21)=2.88$ (higher than the $t$ critical value of 2.09), $p<.01$.

Table 4: Regression Analysis: Impact of Efficacy Ratings of Individual Emotions on Effectiveness of ERS for ASD Individuals. P-value of both "fear" and "happiness" is less than 0.05 making them factors in the equation.

\begin{tabular}{|l|r|}
\hline \multicolumn{2}{|c|}{ Regression Statistics } \\
\hline Multiple R & $\mathbf{0 . 8 5 8 9 1 7 3 7 9 5}$ \\
\hline R Square & 0.7377390648 \\
\hline Adjusted R Square & 0.6503187531 \\
\hline Standard Error & 0.6634124299 \\
\hline Observations & 29 \\
\hline
\end{tabular}

\begin{tabular}{|c|c|c|c|c|c|}
\hline \multicolumn{6}{|c|}{ ANOVA } \\
\hline & df & SS & MS & $F$ & Significance F \\
\hline Regression & 7 & 25.99894222 & 3.714134602 & 8.438989181 & $\begin{array}{r}0.0000627063159 \\
5\end{array}$ \\
\hline Residual & 21 & 9.242437095 & 0.4401160521 & & \\
\hline Total & 28 & 35.24137931 & & & \\
\hline
\end{tabular}

\begin{tabular}{|l|l|l|l|l|l|l|l|l|}
\hline & Coefficients & $\begin{array}{l}\text { Standar } \\
\text { d Error }\end{array}$ & $\mathbf{t}$ Stat & P-value & $\begin{array}{l}\text { Lower } \\
\mathbf{9 5 \%}\end{array}$ & $\begin{array}{l}\text { Upper } \\
\mathbf{9 5 \%}\end{array}$ & $\begin{array}{l}\text { Lower } \\
\mathbf{9 5 . 0} \%\end{array}$ & $\begin{array}{l}\text { Upper } \\
\mathbf{9 5 . 0 \%}\end{array}$ \\
\hline Intercept & 0.9012057185 & 0.830615 & 1.08498524 & 0.29022733 & 0.82615407 & 2.62856551 & 0.82615407 & 2.62856551 \\
\hline Anger & 0.1129709058 & 0.171240 & 0.65972061 & 0.51660381 & 0.46908507 & 0.24314326 & 0.46908507 & 0.24314326 \\
\hline Disgust & 0.0480253587 & 0.105200 & 0.45651261 & 0.65270794 & 0.26680178 & 0.17075107 & 0.26680178 & 0.17075107 \\
\hline Fear & 0.5747291244 & 0.199304 & 2.88367880 & 0.00888696 & 0.16025347 & 0.98920477 & 0.16025347 & 0.98920477 \\
\hline Happiness & 0.701466054 & 0.218934 & 3.20400308 & 0.00426372 & 0.24616733 & 1.15676477 & 0.24616733 & 1.15676477 \\
\hline Sadness & 0.3634688226 & 0.178930 & 2.03133762 & 0.05506861 & 0.73557574 & 0.00863809 & 0.73557574 & 0.00863809 \\
\hline Surprise & 0.0321824425 & 0.139747 & 0.23028946 & 0.82009537 & 0.32280386 & 0.25843898 & 0.32280386 & 0.25843898 \\
\hline Neutrality & 0.0722857987 & 0.124007 & 0.58291293 & 0.56615950 & 0.18560271 & 0.33017430 & 0.18560271 & 0.33017430 \\
\hline
\end{tabular}

A limited regression analysis was done again with the statistically significant variables - happiness and fear. The predictive effect of happiness and fear was confirmed (see Table 5): happiness: $b=0.59, t(26)=4.53$ (higher than the $t$ critical value of 2.06), $p<.01$; and fear: $b=0.28, t(26)=2.67$ (higher than the critical value of 2.06), $p=.013$.
Essentially, the rating given to interpretation of happiness and fear aggregated accounted for about $65 \%$ of the efficiency rating that the respondents give to the program.

Table 5: Impact of Ratings of Happiness and Fear on Overall Efficiency Rating of the Software. Coefficients of "fear" and "bappiness" (significant factors) are 0.28 and 0.59 respectively.

\begin{tabular}{|l|r|}
\hline Regression Statistics \\
\hline Multiple R & 0.807359643 \\
\hline R Square & 0.651829593 \\
\hline Adjusted R Square & 0.625047254 \\
\hline Standard Error & 0.686966727 \\
\hline Observations & 29 \\
\hline
\end{tabular}

\begin{tabular}{|l|r|c|c|c|r|}
\hline \multicolumn{7}{|c|}{ ANOVA } \\
\hline & $d f$ & \multicolumn{1}{c|}{$\boldsymbol{S S}$} & $\boldsymbol{M S}$ & $\boldsymbol{F}$ & Significance $\boldsymbol{F}$ \\
\hline Regression & 2 & 22.97137392 & 11.48568696 & 24.33803828 & $1.10 \mathrm{E}-06$ \\
\hline Residual & 26 & 12.27000539 & 0.471923284 & & \\
\hline Total & 28 & 35.24137931 & & & \\
\hline
\end{tabular}

\begin{tabular}{|l|c|c|c|c|c|c|c|c|}
\hline & Coefficients & $\begin{array}{c}\text { Standard } \\
\text { Error }\end{array}$ & $\boldsymbol{t}$ Stat & $\boldsymbol{P}$-value & Lower 95\% & Upper 95\% & $\begin{array}{c}\text { Lower } \\
95.0 \%\end{array}$ & $\begin{array}{c}\text { Upper } \\
\mathbf{9 5 . 0 \%}\end{array}$ \\
\hline Intercept & 0.327706267 & 0.757296392 & 0.432731848 & 0.668778009 & 1.228938745 & 1.884351279 & 1.228938745 & 1.884351279 \\
\hline Fear & 0.282127031 & 0.105609104 & 2.67142717 & 0.012859088 & 0.06504441 & 0.499209652 & 0.06504441 & 0.499209652 \\
\hline Happiness & 0.592295609 & 0.130790391 & 4.528586568 & 0.000116662 & 0.323452112 & 0.861139105 & 0.323452112 & 0.861139105 \\
\hline
\end{tabular}

The linear equation to calculate the extent of the impact of the efficacy ratings of these two emotions on the effectiveness rating of the ERS was as follows:

Effectiveness of ERS $=0.33+0.59^{*}$ (efficacy rating Happiness $)+$ $0.28^{*}$ (efficacy rating Fear)

For example, if an individual were to rate the efficacy rating of happiness at "7", and fear as " 6 ", then his/her likely rating of the overall effectiveness of the software would be 6.14 out of 7. Conversely, if an individual were to rate the efficacy rating of happiness at "1", and fear as "2", then his/her likely rating of the overall effectiveness of the software would be 1.48 out of 7. If an individual were to rate the efficacy rating of happiness at " 5 ", and fear as " 4 ", then his/her likely rating of the overall effectiveness of the software would be 4.04 out of 7 using the equation. These results highlight the importance of these two factors that account for $65 \%$ of the impact. Nonetheless, there still are other factors that could be identified to help determine the effectiveness of this video and the self-designed ERS in demonstrating its viability for ASD individuals to people who interact with them.

\section{Conclusion}

The research study set out to evaluate the impact of a selfformulated video on a self-designed ERS on the perceptions of autism professionals' or family members of the effectiveness of ERSs as a tool to ASD individuals to interpret facial expressions in daily interactions. All the null hypotheses set out in this research study were rejected due to the statistical significance of the analyses. The results showed that the video featuring a self-designed ERS had a positive effect on the respondents' opinions on the effectiveness of the ERS for people with ASD. The respondents started believing that such a tool could make lives easier which was reflected in a 15\% increase in their ratings of the ERS' effectiveness after watching the video. There were differences between the mean ratings of the peoples' perception of the software's efficiency of interpreting different emotions, 
which were better understood through the analysis of qualitative responses. Some emotions were clearly identified without ambiguity (like happiness, sadness and fear), whereas others were ambiguous (like anger, disgust, and neutrality). Finally, it was found that only happiness and fear out of all the seven emotions had an effect on the respondents' rating on the ERS' effectiveness, which makes sense because they both are easily identified by neurotypicals themselves and have a high mean rating.

While the respondents gave slightly above average to high ratings of the efficacy of individual emotions and the effectiveness of the ERS for ASD individuals, they also pointed out the challenge of this objective. Respondents suggested that their efficacy ratings for different emotions could be biased by their opinions of the ambiguity present. For example, one respondent commented that "the facial expressions for disgust, fear and sadness seem too similar." This means that even neurotypicals struggle to judge certain categories of emotion. In fact, others pointed out that the ERS should include more features like "complex emotions" like "sarcasm", which can be ambiguous, and "condescending behavior", as well as additional features like "body language", to help ASD individuals in interactions. One respondent said, "Some emotions will never be visible." This suggested that there is still a need to generate support for ERS as well as trying to make parents and professionals understand its working better. A good way to do it, as suggested by the respondents, would be by having a way to justify choosing one emotion over the other since neurotypicals themselves get confused with emotions and the only way their brain decides which is which is by providing reason. This might even make the program more relatable for them. It is important to note that these suggestions might make up for the remaining $35 \%$ constituent factors or emotions that affect perception of respondents on the viability of the ERS.

Respondents are inclined to give the ERS a try since they already approve of its working principle, that is, it is a visual aid. They wrote about visual aids being a common means in helping the problems of individuals with ASD. One respondent shared that their son/sibling benefited from it. One of them talked about a similar concept saying, "There is something similar in the US where the child wears spectacles that interpret the emotions being observed." This is similar to the Google glass concept discussed in the introduction. ${ }^{16}$ Much like the spectacle example above, there are various ways to harness this program in real life, some of them being through physical tools, in the form of a mobile or tablet application, or by using it interlinked with video conferencing platforms like Zoom and Google meet. This tool being available not only during face-toface interactions, but also during virtual meets, could magnify the potential of its helpfulness for people diagnosed with ASD, with the increase of work from home culture in today's in progressive world. ${ }^{19}$ While neurotypicals are chasing efficiency, this tool can help individuals with ASD to maintain, if not improve, their current comfort during interactions.

However, this innovation should not stop here. According to the quantitative data, only the respondents' efficacy ratings of the emotions of happiness and fear predicted their rating on the overall effectiveness of the software. These two emotions together accounted for $65 \%$ of the respondents' perception of the software as an effective tool for ASD individuals. This means that the other five emotions do not affect the decision of the respondents effectively, which points towards another opportunity for improvement: the ERS' recognition algorithm and display, and possibly using other ways to interpret emotions, that is, through tone or voice.

Based on the concerns raised by the respondents, the functioning of the ERS as a helpful interaction tool for ASD individuals can possibly be augmented by integrating it with a kind of sound recognition tool. Through the medium of sound and tone, this tool would supplement the evaluation of facial recognition by generating its own output so that a more accurate outcome can be produced. Various ways of integrating the two systems can be explored, working simultaneously, working on the added probabilities from both systems, working alternately, etc. Since facial expressions are not the only thing that determines emotions, such a system could help the computer minimize the error, or ambiguity in the interpretation of facial expressions and give a more reliable prediction of emotions.

\section{References}

1. Kanner, L. (1943). Autistic disturbances of affective contact. Nervous Child,2(3), 217-250.http://mail.neurodiversity.com/ library_kanner_1943.pdf

2. Faras, H., Al Ateeqi, N., \& Tidmarsh, L. (2010). Autism spectrum disorders. Annals of Saudi Medicine, 30(4), 295-300. https://doi. org/10.4103/0256-4947.65261

3. Baron-Cohen, S. (1989). The autistic child's theory of mind: A case of specific developmental delay. Journal of Child Psychology and Psychiatry, 30(2), 285-97.

https://doi.org/10.1111/j.1469-7610.1989.tb00241.x

4. Baron-Cohen, S., Jolliffe, T., Mortimore, C., \& Robertson, M. (1997). Another advanced test of theory of mind: evidence from very high functioning adults with autism or Asperger syndrome. Journal of Child Psychology and Psychiatry, 38, 813-822. https://doi.org/10.1111/j.1469-7610.1997.tb01599.x

5. Dalton, K. M., Nacewicz, B. M., Johnstone, T., Schaefer, H. S., Gernsbacher, M. A., Goldsmith, H. H., Alexander, A. L., \& Davidson, R. J. (2005). Gaze fixation and the neural circuitry of face processing in autism. Nature Neuroscience, 8(4), 519-526. https://doi.org/10.1038/nn1421

6. Klin, A., Jones, W., Schultz, R., Volkmar, F., \& Cohen, D. (2002). $\mathrm{Visual}$ fixation patterns during viewing of naturalistic social situations as predictors of social competence in individuals with autism. Archives of General Psychiatry, 59(9), 809-816. https://doi. org/10.1001/archpsyc.59.9.809

7. Volkmar, F. R., \& Mayes, L. C. (1990). Gaze behavior in autism. Development and Psychopathology, 2(1), 61-69. https://doi. org/10.1017/S0954579400000596

8. Leekam, S. R., López, B., \& Moore, C. (2000). Attention and joint attention in preschool children with autism. Developmental Psychology, 36(2), 261-273.

https://doi.org/10.1037//0012-1649.36.2.261

9. Grossman, R. B., Bemis, R. H., Skwerer, D. P., \& Tager-Flusberg, H. (2010). Lexical and affective prosody in children with highfunctioning autism.Journal of Speech, Language, and Hearing Research : JSLHR. 53. 778-93.https://doi.org/10.1044/1092-4388

10.Baron-Cohen, S., Wheelwright, S., Spong, A., Scahill, V., \& Lawson, J.(2001). Are intuitive physics 
and intuitive psychology independent? A test with children with Asperger Syndrome. Journal of Developmental and Learning

Disorders, 5. https://docs.autismresearchcentre.com/papers/2001_ BCetal_kidseyes.pdf.

11.Golan, O., Sinai-Gavrilov, Y., \& Baron-Cohen, S. (2015). The Cambridge Mindreading Face-Voice Battery for Children (CAM-C): Complex emotion recognition in children with and without autism spectrum conditions. Molecular autism, 6, 22. https://doi.org/10.1186/s13229-015-0018-z

12.Hopkins, I. M., Gower, M. W., Perez, T. A., Smith, D. S., Amthor, F. R., Wimsatt, F. C., \& Biasini, F. J. (2011). Avatar assistant: improving social skills in students with an ASD through a computer-based intervention. Journal of Autism and Developmental Disorders, 41(11), 1543-1555. https://doi.org/10.1007/s10803-011-1179-z

13.LaCava, P. G., Rankin, A., Mahlios, E., Cook, K., \& Simpson, R. L. (2010). A single case design evaluation of a software and tutor intervention addressing emotion recognition and social interaction in four boys with ASD. Autism: The International Journal of Research and Practice, 14(3), 161-178. https://doi.org/10.1177/1362361310362085

14.Simmons, E. S., Paul, R., \& Shic, F. (2016). Brief report: A mobile application to treat prosodic deficits in autism spectrum disorder and other communication impairments: A pilot study. Journal of Autism and Developmental Disorders, 46(1), 320-327. https://doi.org/10.1007/s10803-015-2573-8

15.Silver, M., \& Oakes, P. (2001). Evaluation of a new computer intervention to teach people with autism or Asperger syndrome to recognize and predict emotions in others. Autism: The International Journal of Research and Practice, 5(3), 299-316. https://doi. org/10.1177/1362361301005003007

16.Washington, P., Voss, C., Haber, N., Tanaka, S., Daniels, J., Feinstein, C., ... \& Wall, D. (2016, May). wearable social interaction aid for children with autism. In Proceedings of the 2016 CHI Conference Extended Abstracts on Human Factors in Computing Systems. https://doi.org/10.1145/2851581.2892282

17.Wu, J. (2017). Introduction to convolutional neural networks. National Key Lab for Novel Software Technology, 5, 23. https://cs.nju.edu.cn/wujx/paper/CNN.pdf

18.Maklin, C. (2019, June 3). Dropout neural network layer in keras explained. Towards data science. https://towardsdatascience.com/ machine-learning-part-20-dropout-keras-layersexplained-8c9f6dc4c9ab

19.Saltiel, F. (2020). Who can work from home in developing countries. Covid Economics, 7(2020), 104-118. http://econweb.umd. edu/ saltiel/files/wfh_mostrecent.pdf

\section{Author}

Aryan Dawer is a 12th grader at Modern School Barakhamba Road, New Delhi, India. He is 17 years old, and very interested in Computer Science and Psychology. His passion for designing tools found purpose in his work with individuals with ASD. Previously, he has designed software to recognize emotions through tone and facial expressions to aid them in daily conversations. He is also an environmentalist and is founding member and CTO of DropCount, a water-saving initiative that uses innovative technology to save water. In his free time, Aryan likes to analyse tv show/movie/novel characters, and put himself in their shoes to enhance his logical reasoning skills as well as experience diverse situations. 